1 Neural Modulators of Temperament: a multivariate approach to personality trait

2 identification in the horse

3

4 Roberts, Kirsty ${ }^{1}$., Hemmings, Andrew.J. ${ }^{1}$, Moore-Colyer, Meriel ${ }^{1}$., Parker, Matthew.O. ${ }^{2}$

5 \& McBride, Sebastian.D. ${ }^{3}$

$6{ }^{1}$ Royal Agricultural University, Stroud Road, Cirencester, Gloucestershire, GL7 6JS

$7{ }^{2}$ School of Health Sciences and Social Work, University of Portsmouth, James Watson

8 West building, 2 King Richard 1st Road, Portsmouth, Hampshire, PO1 2FR

$9{ }^{3}$ Aberystwyth University, Penglais, Aberystwyth, Ceredigion, SY23 3DA

11 Corresponding Author: K. Roberts $\quad$ Tel: $01285 \quad 652531$.

\title{
12 kirsty.roberts@student.rau.ac.uk
}

14 Ethics: Ethical approval was provided by the ethics committee and the Royal 15 Agricultural University

\section{ABSTRACT}

18 A relationship between dopamine and temperament has previously been described in

19 human cases of dopaminergic dysfunction. Adjustment in temperament prior to

20 disease manifestation can enable the early identification of individuals at risk of such

21 conditions, and scope exists to extend this application of temperament alterations to 
cases of dopaminergic dysfunction in horses. A multivariate and mixed-methods

approach utilising a questionnaire along with two inferred measurements of dopamine activity (Spontaneous Blink Rate [SBR] and Behavioral Initiation Rate [BIR]) were recorded from direct observation of animals $(n=99)$ to identify the potential relationship between dopamine and temperament in horses. Principal components analysis (PCA) of 36 temperament variables revealed nine Principal Components, including 'Anxiety' and 'Docility', which accounted for $72.4 \%$ of the total variance. Component scores were calculated and correlated with SBR and BIR utilising Spearman Rank Correlation Coefficient analysis. The component 'Anxiety' was found to have a significant positive relationship with SBR, whereas 'Docility' was observed to have a significant negative relationship with SBR. These results indicate a relationship between dopamine and temperament within the horse that is certainly worthy of further study. Potential mechanisms involving neural dopaminergic and GABAergic systems are presented, in addition to how such alterations could be utilised to probe for equine dopamine dysfunction pending future research.

Highlights

- A significant positive correlation was observed between 'Anxiety' and dopamine levels

- A significant negative correlation was found between 'Docility' and dopamine levels

- Chronic dopamine adaptations may initially manifest as temperament alterations

- Potential exists to identify horses at risk of dopamine dysfunction development through analysis of temperament Key words: Temperament; Dopamine; Equine; Behavior; Anxiety; Docility 


\subsection{Introduction}

47 Equine temperament is defined as any characteristic of an individual which emerges at a young age and appears to be stable, over both time and situation (Lansade \& Simon, 2010). However, this could neglect the distinct behavioral manifestation of temperament. Indeed, Stur (1987) suggested temperament is the sum of inherited and learned behavior patterns, whilst Kilgour (1975) highlights the distinct physical, hormonal and nervous characteristics of an animals' temperament. It is the combination of temperament and environmental conditions which are proposed to amalgamate as equine personality (see Randle, 2015). Temperament is of importance for the performance and leisure horse, for competition success as well as improving equine welfare and understanding (Visser et al., 2001; Randle, 2015). It is perhaps for this reason that a plethora of temperament research does exist for the horse, utilising both questionnaire and behavior methodologies (Seaman et al., 2002; Momozawa et al., 2003; Visser et al., 2008). Whilst a number of studies have investigated learning and memory functioning in horses in relation to temperament traits (Lansade \& Simon, 2010; Lansade et al., 2013; Valenchon et al., 2013), the way in which this relates to neural functioning has not been investigated. This is perhaps surprising given Kilgour's (1975) early reference to

Of particular interest to date is the relationship between the neurotransmitter dopamine (DA) and its role in temperament manifestation, with specific reference to human conditions of DA dysfunction, such as Parkinson's disease (PD), a hypodopaminergic condition (Burch \& 
personality which can predate the emergence of motor symptoms, in some instances by decades (Todes \& Lees, 1985). Indeed, PD patients often present with stoic, rigid and slow temperaments as a part of this characteristic parkinsonian personality (Dagher \& Robbins, 2009), whilst also demonstrating low novelty seeking scores (Kaasinen et al., 2001). This alteration in temperament has previously been linked to the hypodopaminergic characteristic of PD, though some conflicting evidence does remain (Kaasinen et al., 2001).

In contrast, patients who exhibit schizophrenia, a hyperdopaminergic condition (Breier et al., 1997) demonstrate an increased prevalence for anxiety disorders (Pallanti et al., 2004; Achim et al., 2009). Moreover, anxiety is thought to contribute as part of the prodromal stage of schizophrenia (Park et al., 2016). When considered in the context of the proposed mechanism of anxiogenic drugs which ultimately elevates neural DA levels (Cancela et al., 2001), this suggests that chronic DA reduction or elevation can manifest as a significantly altered temperament.

Horses can also present with hyper- and hypo-dopaminergic conditions, including stereotypic behaviours (SB; McBride \& Hemmings, 2005; Roberts et al., 2015) and pituitary pars intermedia dysfunction (PPID; McFarlane et al., 2005), respectively. Interestingly, there is also an observation that horses with PPID present with significant alterations in temperament, including an increase in depression, lethargy and apathetic manifestations with disease progression (Bradaric et al., 2013). However, this temperament alteration has not yet been linked with the reduction of DA which occurs within these animals. Furthermore, in the light of the pre-morbid personality associated with PD and anxiety within the prodromal phase of schizophrenia, it is plausible that dopaminergic alterations could manifest prior to 
conventional diagnosis through analysis of temperament traits. This approach would however need to proceed mindful of the balance between genotypic determination of temperament and subsequent environmental modification of behavioural output. Whilst temperament and personality type research has been conducted in horses (for example see Momozawa et al., 2003; Momozawa et al., 2005a; Lloyd et al., 2007, Nagy et al., 2010; ljichi et al., 2013), the way in which the result of this relates to dopaminergic function has yet to be determined empirically. Interestingly however, Momozawa et al. (2005b) observed a significant relationship between DRD4 (dopamine D4 receptor subtype) single nucleotide polymorphism, an A-G substitution causing an amino acid change from asparagine to aspartic acid (Hori et al., 2013). Absence of the 'A' allele resulted in significantly increased 'Curiosity' and lower 'Vigilance' scores than those with the 'A' allele (Momozawa et al., 2005b). Whilst persuasive of an influence of dopamine over temperament in the horse, the precise mechanisms as to how the $D R D 4$ gene directly influences temperament in any species is currently unknown and requires further research.

Previous research has demonstrated the successful utilisation of the proven DA correlates spontaneous blink rate (SBR) and behavioral initiation rate (BIR) to investigate dopaminergic function in oral and locomotor stereotypy in the horse (Roberts et al., 2015), but as of yet no links have been made to any temperament data. It is for this reason that SBR and BIR were utilised here to examine the role of dopamine in the generation of temperament. A questionnaire was used to gather temperament data due to previous validation of this approach within the literature (Momozawa et al., 2003; Momozawa et al., 2005a; Lloyd et al., 2007). Results of this study could provide a valuable basis for the early identification of horses undergoing initial DA dysfunction thereby improving welfare through timely intervention. 


\subsection{Methods}

\subsection{Sample Population Characteristics and Management}

A sample of 100 horses of varying backgrounds were sought, comprising 52 geldings, 44 mares and four stallions, aged 2-25 years (mean \pm SD $12.17 \pm 6.24$ years) and included some stereotypy performing horses ( $n=3$ crib-biters, $n=15$ weavers, $n=9$ box walkers) due to previously described linkage between SB, dopamine (Cabib \& Bonaventura, 1997) and temperament traits (Dagher \& Robbins, 2009; Park et al., 2016). All animals were recruited through direct contact from private owners $(n=40)$, and were kept at various locations in Gloucestershire and Wiltshire, in the South West of England during the study period (June 2014-January 2015). In addition, these animals differed in terms of management regime, breed (ranging from native breeds to sport horses) and use (from leisure to polo and competition disciplines such as polo and dressage) in order to source a representative crosssection of the UK horse population with the level of variance favoured for Principal Component Analysis (ljichi et al., 2013). As such, selection criteria required that the horse was free from acute clinical disease (excluding dopamine pathology) and able to be led from a head collar and lead rope (ljichi et al., 2013).

\subsection{Equine Temperament Questionnaire}

Owners were asked to complete a two-part questionnaire. The first section of the questionnaire was designed to collect information regarding the management and feeding regime of each individual, along with factors (i.e. breed) shown by other studies to impact upon temperament (Hausberger \& Muller, 2002; Lloyd et al., 2008). Section 2 was based on 
141 previous equine temperament questionnaires (Momozawa et al., 2003; Momozawa et al., 142 2005a; Lloyd et al., 2007). A 1-9 Likert scale was used to assess 41 temperament descriptions. Each description was identified by a definition in order to ensure that participants had an agreed understanding of the temperament term being used. For example the temperament description 'Concentration' was accompanied by the definition 'is trainable and undisturbed by the environment'. All of the chosen temperament descriptions were selected from the aforementioned studies, though some were omitted due to repetition or irrelevance with reference to dopamine. One additional temperament description 'Impulsive' with accompanying definition 'tends to act without forethought, regardless of the consequences' was added, as impulsivity is thought to be characteristic of abnormal behaviors such as stereotypy (Garner \& Mason, 2002) and has links back to dopamine transmission (Cools, 2008).

\subsection{Spontaneous Blink Rate Observation}

The method to assess SBR for all of the horses has previously been described in Roberts et al. (2015). To reduce stress for the animal during observations, all horses had visual contact with conspecifics. The purpose of this was for both ethical reasons i.e. not to isolate a social species, but also to avoid artificially inducing alterations in the horses SBR due to external factors. Each horse was stabled in their home stable and habituated to the researcher's presence by having the researcher stand calmly outside the stable with the horse in full view for 10 minutes prior to the observation period. Following the 10 minute habituation period, continuous method sampling was utilised where each full blink (defined by Karson (1983) as bilateral paroxysmal brief repetitive eye closures occurring continuously) was recorded with a mechanical counter for 30 minutes. Considering the anatomy of the horse, it was difficult 
for a solo observer to record true bilateral eye closures, so only the left eye was observed for all horses. As such, the horses head collar remained on throughout to ensure the horse remained in position in such a way that the observer had full view of the horses left eye whilst outside the stable at all times. This procedure was repeated in the same manner over three consecutive days, allowing a mean SBR/30minutes to be calculated for each horse.

\subsection{Behavioral Initiation Rate Observation}

This procedure has previously been described for use in the horse by Roberts et al. (2015), based on BIR observations of bank voles (Garner \& Mason, 2002). The horse was stabled within their home stable and habituated to the observers' presence for 10 minutes as described in Section 2.3. Following habituation, each behavioral initiation was recorded by continuous sampling utilising a mechanical counter for 30 minutes where the observer was positioned outside of the stable though with full view of the horse. All behaviors performed were defined by a pre-determined ethogram (McDonnell, 2003), and similar to Garner and Mason (2002) only the number, not the type, of behaviors was recorded. Furthermore, each bout of behavior was recorded as one initiation irrespective of the previous behavior, consequently the sequence 'Feeding - Grooming - Feeding - Drinking - Standing Rest' was recorded as four initiations (Garner \& Mason, 2002; Roberts et al., 2015). Thus, movement made as part of an identifiable behavior, for example lifting of the head whilst still undergoing mastication as part of feeding was accepted as part of the behavior and therefore not recorded as an initiation of a new behavior (Roberts et al., 2015). Recording of stereotypic behaviors was considered in terms of bouts of behavior, i.e. regardless of the number of weaves produced, each single bout of weaving was recorded as one behavioral initiation. 
BIR recording was repeated over three consecutive days in the same manner, allowing a mean BIR/30minutes to be calculated for each horse.

\subsection{Statistical Analysis}

192

A PCA was carried out with varimax rotation on all questionnaire items. Components with eigenvalues $\geq 1$ were retained. Component loadings of $\geq 0.4$ within each component were considered significant (McGrogan et al., 2008) and checked for PCA suitability with the Kaiser-Myer-Olkin (KMO) statistic. The Anderson-Rubin method for standardising component scores was applied to ensure that all component scores were directly orthogonal. Once component scores had been calculated for every horse for each temperament component, these were then correlated with the horses' mean SBR and BIR utilising a Spearman Rank Correlation Coefficient, similar to that conducted previously utilising both behavioral and questionnaire measures (Lloyd et al., 2007). Mean SBR and BIR values were also calculated. A second PCA was conducted to investigate SBR and BIR as contributory towards component structure i.e. if for example SBR contributed towards a particular temperament component. To examine if sex, breed and usage had an influence on temperament and dopamine correlate data, Kruskal-Wallis with post-hoc pairwise comparisons and Bonferroni correction, or Mann-Whitney $U$ tests where appropriate, were undertaken. To decipher whether median or mean-rank was presented, visual inspection of the box-plots produced via SPSS were utilised. Where distributions were similar median values were presented, though when distributions were dissimilar, median-ranks were utilised. Breeds were categorised into pony/cob types (14.2hh and under), sport horse types (light breeds e.g. Thoroughbreds 14.3hh and over) and draught horse types (heavy breeds e.g. Irish Draught 14.3hh and over). 
212 jumping and cross-country jumping) or polo dependent on the horse's primary purpose. The significance level was set at $p<0.05$. All statistical analyses were conducted in IBM SPSS version 22.

\subsection{Results}

\subsection{PCA Data}

A $99 \%$ return rate was achieved from the questionnaires, only animals with which temperament data were available were utilised for subsequent analysis, giving a total of $n=99$. Initially, all 41 temperament variables were utilised to perform the PCA. Five temperament traits (Timidity, Sociable, Protective, Subordinate and Permissive) were deemed unreliable following examination of the KMO statistic and were consequently removed from further PCA analysis. When the PCA was conducted with the remaining 36 temperament descriptions, nine components were extracted accounting for $72.4 \%$ of the total variance (see Table 1 ). The names of the components were derived from examination of the individual temperament definitions which contribute towards a component (Table 2). Previous research was also taken into account (Momozawa et al., 2003; Momozawa et al., 2005; Lloyd et al., 2007; Nagy et al., 2010; ljichi et al., 2013; See Appendix 1). As such, the components were named as follows, in descending order of percentage variance explained; 'Anxiety', 'Trainability', 'Excitability', ‘Docility', 'Inquisitiveness', 'Irritability’, 'Self-Reliance', 'Horse-Horse Interaction’ and 'Horse-Human Interaction'. 
Table 1. The total variance explained by the 9 extracted components

\begin{tabular}{lcllll}
\hline Component Name & $\begin{array}{c}\text { Component } \\
\text { Number }\end{array}$ & Total & Variance (\%) & Cumulative (\%) \\
\hline Anxiety & 1 & 11.415 & 31.708 & 31.708 & 236 \\
Trainability & 2 & 4.058 & 11.274 & 42.981 & 236 \\
Excitability & 3 & 2.418 & 6.716 & 49.697 & \\
Docility & 4 & 2.027 & 5.631 & 55.329 & 237 \\
Inquisitiveness & 5 & 1.596 & 4.433 & 59.762 & \\
Irritability & 6 & 1.302 & 3.617 & 63.379 & \\
Self-Reliance & 7 & 1.131 & 3.143 & 66.522 & 238 \\
Horse-Horse Interaction & 8 & 1.073 & 2.981 & 69.503 & \\
Horse-Human Interaction & 9 & 1.049 & 2.913 & 72.415 & \\
\hline
\end{tabular}

239

240 The mean $( \pm$ SEM) SBR/30 min was found to be $547.72 \pm 15.66$, whilst the mean $( \pm S E M)$

$241 \mathrm{BIR} / 30$ min was $24.94 \pm 2.30$. The lowest value for $\mathrm{SBR} / 30$ min was 243 , whilst the highest

242 was 1140, whereas the smallest value for BIR/30 min was 1 though the highest was 133 . The

243 Spearman rank correlation coefficient analyses determined that the temperament component

244 'Anxiety' was positively correlated with SBR $\left(r_{s}(97)=0.202, p=0.045\right)$ and 'Docility' was

245 negatively correlated with $\operatorname{SBR}\left(r_{s}(97)=-0.215, p=0.032\right)$. There were no significant

246 correlations between temperament traits and BIR.

Table 2. The component loadings for each of the 9 extracted components. Component 1, 24hkiety; Component 2, Trainability; Component 3, Excitability; Component 4, Docility; Component 5, Inquisitiveness; Component 6, Irritability; Component 7, Self-Reliance; Component 8, Horse-Horse Interaction; Component 9, Horse-Human Interaction.

\begin{tabular}{|c|c|c|c|c|c|c|c|c|c|}
\hline \multirow{2}{*}{ Temperament Trait } & \multicolumn{9}{|c|}{ Component } \\
\hline & 1 & 2 & 3 & 4 & 5 & 6 & 7 & 8 & 9 \\
\hline Nervousness & .820 & & & & & & & & \\
\hline Concentration & & .647 & & & & & & & \\
\hline Self_reliance & & & -.434 & & & & .644 & & \\
\hline Trainability & & .793 & & & & & & & \\
\hline Excitability & .528 & & .499 & & & & & & \\
\hline Friendliness_people & & & & & & & & & .859 \\
\hline Friendliness_horse & & & & & & & & .777 & \\
\hline Curiosity & -.452 & & & & .402 & & & & \\
\hline Memory & & .816 & & & & & & & \\
\hline Panic & .768 & & & & & & & & \\
\hline Cooperation & & .636 & & & & & & & \\
\hline Inconsistent & .481 & & & & & .582 & & & \\
\hline
\end{tabular}




\begin{tabular}{|c|c|c|c|c|c|c|c|c|c|}
\hline Stubborness & & & & & & .665 & & & \\
\hline Docility & & & & .766 & & & & & \\
\hline Vigilance & & .440 & & & .421 & & & & \\
\hline Patience & & .406 & & .588 & & & & & \\
\hline Competitiveness & & & & & & & & -.632 & \\
\hline Skittishness & .687 & & & & & & & & \\
\hline Active & & & .595 & -.409 & & & & & \\
\hline Impulsive & .447 & & .675 & & & & & & \\
\hline Apprehensive & .767 & & & & & & & & \\
\hline Confident & -.660 & & & & & & & & \\
\hline Eccentric & & & .583 & & & & & & \\
\hline Equable & -.416 & & & .522 & & & & & \\
\hline Fearful & .855 & & & & & & & & \\
\hline Irritable & & & & & & .536 & & & -.447 \\
\hline Opportunistic & & & & & .556 & & & & \\
\hline Playful & & & & & .752 & & & & \\
\hline Popular & & & & & & & & .550 & \\
\hline Slow & & & & .708 & & & & & \\
\hline Solitary & & & & & & & .812 & & \\
\hline Tense & .686 & & & & & & & & \\
\hline Suspicious & .776 & & & & & & & & \\
\hline Reliable & & .417 & & & & & & & \\
\hline Hardworking & & .775 & & & & & & & \\
\hline Intelligent & & .706 & & & & & & & \\
\hline
\end{tabular}

249 Whilst the second PCA resulted in the dopamine correlates contributing to their own 250 component, analysis of the KMO statistics revealed that neither SBR nor BIR were suitable 251 for PCA $(p<0.05)$. The PCA was disregarded due to the unreliability of SBR and BIR as 252 contributory variables to overall temperament components.

255 Distributions of temperament traits and dopamine correlates were similar as assessed by 256 visual inspection. Median 'Anxiety' score for mares $(n=44 ;-0.54)$ versus geldings $(n=55 ; 0.05)$ 257 were significantly different $(U=1534, z=2.282, p=0.023)$. Furthermore, median 258 'Inquisitiveness' scores for mares $(-0.23)$ was significantly lower than geldings $(0.09)(U=$ 
1489, $z=1.965, p=0.049)$. Likewise, median 'Horse-Human Interaction' scores were significantly lower for mares $(-0.13)$ than geldings $(0.39)(U=1654, z=3.127, p=0.002)$. No other significant differences were observed between mares and geldings for temperament components or dopamine correlates.

\subsection{Effect of Breed}

Distributions of temperament and dopamine correlates were not similar for pony/cob types $(n=33)$, sport horse types $(n=60)$ and draught types $(n=6)$ as assessed by visual inspection of the box-plot, thus mean-ranks are provided. SBR $\left(X^{2}(2)=6.872, p=0.032\right)$, 'Anxiety' $\left(X^{2}\right.$ $(2)=8.665, p=0.013)$ and 'Excitability' $\left(X^{2}(2)=12.916, p=0.002\right)$ scores were significantly different between the three breed categories when corrected for ties. Pairwise comparisons with Bonferroni correction revealed significant differences in SBR between pony/cob types (39.35) against sports horse types $(55.61 ; p=0.027)$ though no difference was found between draught types (52.50) against either pony/cobs $(p=0.907)$ or sport horses $(p=1.000)$. Similarly, pony/cob types demonstrated a significantly lower mean rank 'Anxiety' score (39.36) than sport horse types $(56.85 ; p=0.015)$. No difference was observed between draught types $(40.00)$ compared to pony/cob types $(p=1.000)$ or sport horse types $(p=$ 0.512). A significantly lower 'Excitability' mean rank for pony/cob types (38.58) compared to sport horse types $(58.23 ; p=0.005)$ was also observed. There was no difference between draught types (30.50) with either pony/cob types $(p=1.000)$ or sports horse types $(p=0.072)$ and no further differences were observed for BIR nor any of the remaining seven temperament components. 
283 Distributions of temperament and dopamine correlates were similar for leisure $(n=56)$, 284 traditional sports discipline $(n=27)$ and polo $(n=19)$ horses as assessed by visual inspection 285 of the box-plot, thus median values are provided. 'Anxiety' $\left(X^{2}(2)=9.418, p=0.009\right.$ ), 286 'Excitability' $\left(X^{2}(2)=8.138, p=0.017\right)$ and 'Inquisitiveness' $\left(X^{2}(2)=6.002, p=0.05\right)$ were significantly different between the three use categories when corrected for ties. Pairwise comparison with Bonferroni correction highlighted that the leisure animals demonstrated a lower median 'Anxiety' score (-0.21) compared to sport discipline horses $(0.50 ; p<0.009)$. No significant difference was observed for median 'Anxiety' score between polo horses ($0.01)$ and leisure $(p=0.335)$ nor traditional sports discipline animals $(p=1.000)$. Leisure animals demonstrated a lower median 'Excitability' score (-0.36) compared to traditional sports discipline horses $(0.49 ; p=0.019)$. Polo animals median score $(-0.30)$ showed no significant difference between either leisure $(p=1.000)$ or sports discipline horses $(p=0.112)$. Furthermore, leisure animals demonstrated an increased median 'Inquisitiveness' score (0.06) compared to polo horses $(-0.51 ; p=0.049)$, though no difference was apparent between traditional sports discipline horses $(0.09)$ and leisure $(p=1.000)$ or polo animals $(p$ $=0.130)$. No other temperament traits, SBR or BIR were found to demonstrate statistical significance between uses.

\subsection{Discussion}

The combined PCA and correlational analysis supported the hypothesis that one known measure of inferred DA activity (SBR) is correlated with the two temperament components 'Anxiety' and 'Docility', a finding not yet reported in the equine literature, although the range of SBR data gathered is roughly in-line with our previous work (Roberts et al., 2015). SBR is 
correlated with striatal DA levels, with increases indicating higher, and decreases lower, levels of production and release of DA (Kaminer et al., 2011). Therefore, our data suggest that SBR may not only be a potentially useful predictor of central dopamine function in relation to behavioral output and as a risk factor for onset of pathology, but also as a proximate predictor tool. Furthermore, given that SBR does not appear to contribute to temperament traits themselves, this could indicate the suitability of SBR as a potential measure of underlying temperament components as opposed to being a direct causal factor. Temperament and personality have previously been cited as highly influential for horse purchase and breeding, and a need to incorporate an objective temperament assessment identified by Graf et al. (2013). Thus the use of SBR as part of pre-purchase veterinary examination may allude to the temperament of the animal and be beneficial to the horse owning population. Indeed, improved understanding of equine temperament prior to purchase could benefit human safety, given that unanticipated horse behavior was highlighted as a contributory factor in $61 \%$ and $39 \%$ of injuries in children under 15 and adults above 15 respectively (Northey, 2003).

\section{1 'Anxiety' and Dopamine}

Due to the causal links between stress and DA (Cabib et al., 1998), it is possible that anxious horses are more sensitive to environmental stressors, such as restricted feeding or social isolation; common stressors faced by stabled horses (McAfee et al., 2002; Ninomiya et al., 2007). Underlying this increased responsiveness to stress, the anxious individuals may have elevated striatal DA in comparison to less anxious animals kept under the same environmental conditions. This could allow the initiation of active coping in an attempt to gain control over the environment, similar to the elevated dopamine levels observed in the active 
coping DBA mouse strain (Cabib \& Bonaventura, 1997; Cabib \& Puglisi-Allegra, 2012). A similar process could be occurring with the anxious horses, as evidenced by the elevation in SBR in these individuals.

From a mechanistic standpoint, when DA agonists are administered, there is a partnership between the elevation of DA levels and the emergence of behaviors characteristic of anxiety (McCullough \& Salamone, 1992; Cancela et al., 2001). This DA elevation appears to result from GABA disinhibition at the level of the midbrain i.e. the ventral tegmental area (VTA) (Biggio et al., 1990; Nikulina et al., 2005). A similar progression could also be apparent in environmentally induced anxiety. For example in rodents chronic stressors such as restricted feeding, social isolation and restricted locomotion are known to induce mu-opioid proliferation (Nikulina et al., 2005), and therefore resulting in GABA disinhibition, in addition to sensitisation of the dopaminergic pathways (Cabib et al., 1998). Combined, this could indicate a relationship between mu-opioid receptor proliferation and GABAergic disinhibition, perhaps giving rise to elevated DA. These chronic stressors are strikingly similar to those faced by the stabled horse, and induce similar neural adaptations. For example, dopaminergic pathways are suggested to be sensitised within stress-linked conditions of the horse including oral (McBride \& Hemmings, 2005) and locomotor stereotypy (Roberts et al., 2015). Further research indicates mu-opioid receptor density is significantly elevated within horses displaying oral stereotypy at the VTA, as well as dorsal and ventral striatal regions (Hemmings et al., 2006). Thus, it is possible that in the more anxious animals, mu-opioid receptor sensitisation and GABAergic disinhibition giving rise to DA elevation has occurred, or is well underway. This could well be a perpetuating issue given that DA elevation within the amygdala, a structure which also receives dopaminergic input from the VTA and is characteristic for its fear gating and anxiety inducing functions, potentiates anxiety in both 
rodent and human models (Kienast et al., 2008). Additionally, this elevation in dopamine effectively removes inhibitory control of the medial prefrontal cortex over amygdala function (de la Mora et al., 2010) giving rise to prolonged 'Anxiety' type responses. This could therefore provide a mechanism by which elevated 'Anxiety' is observed in this population of horses presenting with raised SBR.

Importantly in rodents, persistent DA elevation is the final step to instigate active coping, leading to stereotypy manifestation in stressed, captive animals (Cabib \& Bonaventura, 1997). The emergence of an elevated 'Anxiety' type temperament in the horse could similarly be indicative of significant underlying neural adaptations which pre-date stereotypy development. Timely removal of key stressors could prevent progression to the stereotypic behavioral end-point in this regard. Indeed, as neural sensitisation of DA appears to be permanent (or at least recalcitrant) where rodent species are concerned, prophylaxis rather than remediation is recommended (Cabib et al., 1998). This latter point is particularly important given the proposed habitual mechanisms of crib-biting behavior (Hemmings et al., 2007; Parker et al., 2009; Roberts et al., 2015), and also with the highly motivated (albeit nonhabitual) phenotype thought to contribute to weaving behavior (Roberts et al., 2015). Also of interest with regard to environmentally induced stereotypy is that under improved management regimes, which reduce chronic stress and potentially the normalisation of DA levels within the neural circuitry, the high 'Anxiety' horses may then become more biddable in the context of training and management. Indeed, the performance of increasingly anxious type behavior is a part of the prodromal phase of schizophrenia (Park et al., 2016) a hyperdopaminergic phenomenon. As such, the use of increasing anxiety is currently under investigation as a potential predictor of an oncoming schizophrenic episode (Park et al., 
2016). In light of this evidence, it is plausible then that the more anxious horses are presenting as such due to consistently elevated levels of the neurotransmitter DA.

However, when investigating the work completed by Nagy et al. (2010), an interesting question arises. It was reported that crib-biting horses demonstrated a significantly lower 'Anxiety' score when compared to the control animals (Nagy et al., 2010). Whilst this initially appears to contradict what is being suggested here, the data reported by Nagy et al. (2010) may in fact lend support. Crib-biting animals present as hypodopaminergic, as evidenced by a significantly decreased SBR and significant reduction in caudate dopamine receptor sensitivity (McBride \& Hemmings, 2005; Roberts et al., 2015). This is despite post-mortem evidence of increased receptor sensitivity to dopamine within the nucleus accumbens (McBride \& Hemmings, 2005). This neural work supports the notion that SBR is a reflection of midbrain DA transmission into the dorsal striatum (caudate nucleus) rather than the ventrally sited nucleus accumbens (Taylor et al., 1999). However, elevations in nucleus accumbens DA are of significant behavioral relevance, and have been considered crucial to the putative self-stimulatory stress reducing aspects of crib-biting. It may be that following the onset of a stress coping function i.e. crib-biting performance, leads to the diminishing anxiety in crib-biting animals as reported by Nagy et al. (2010). Indeed, recent evidence appears to support the stress coping function of crib-biting (Freymond et al., 2015). Overall then, it is plausible that initial elevations in DA could manifest as increased 'Anxiety', though potentially following the development of stereotypy as a part of the behavioral repertoire 'Anxiety' could decrease, at least in crib-biting horses. Further research would be required to investigate such an effect in weaving animals. 


\section{2 'Docility’ and Dopamine}

404 'Docility' was negatively correlated with SBR, and thus inferred levels of DA activity. A 405 comparative phenomenon is observed in the inbred mouse strain C57, whereby in response to an inescapable stressor, a net reduction in DA transmission leads to reduced anxiety linked behaviors such as locomotion (Cabib, 2006; Cabib \& Puglisi-Allegra, 2012). Rather than the often quoted pathological condition of learned helplessness, the depressed activity in the C57 strain is thought to signal a passive form of coping, which promotes energy thriftiness in the face of insurmountable stress (Cabib \& Puglisi-Allegra, 2012). The reduced SBR which accompanies 'Docility' in the data reported herein, could well indicate a similar strategy is adopted by the horse in response to persistent low level stressors such as restricted locomotion and social isolation.

From a pathology prediction standpoint, in stark contrast to those with conditions characterised by elevated DA such as schizophrenia and drug addiction, human patients with PD typically present with personalities which are categorised as stoic, rigid and slowtempered (Dagher \& Robbins, 2009). Novelty-seeking type behaviors are also reduced in PD, with twin studies also demonstrating that individuals who later develop Parkinson's are more self-controlled than their non-effected twin (Menza, 2000). It is thought that the emergence of such traits are directly related to underlying degeneration of DA containing neurons (Kaasinen et al., 2001). When considering the individual temperament variables 'slow' and 'patient' which contribute to the 'Docility' temperament trait in this cohort of horses (Table 2), it is reasonable to propose that the more 'docile' horses are also more slow-tempered and selfcontrolled. Parallels could be drawn between Parkinson's disease personality and conditions of the horse which feature reduced DA such as PPID. Typically, PPID is suspected when 
427 overt symptoms, such as hirsutism and hyperhidrosis are observed in combination with 428 advancing age, at which point it is likely that the influence of elevated proopiomelanocortin (POMC) peptide levels are already having a significant detrimental effect on health (McFarlane, 2011). Indeed, ACTH is characteristically elevated in PPID horses prior to pharmacological treatment, with this posing long term health issues leading to reduced welfare status of the animal (Durham et al., 2014). Early intervention with regards to PPID development could be essential for prolonged quality of life and improved welfare (McFarlane et al., 2011). Despite this, there is a lack of peer reviewed publications specifying the early indicators of PPID, which when treated earlier could significantly improve prognosis and prevent life threatening consequences of the disease (McFarlane et al., 2005). Therefore, our finding that a decrease in SBR is correlated with higher 'Docility' could provide an important early indicator for those at risk of PPID development. This is of significance given that depression, lethargy and an apathetic outlook are observed following PPID diagnoses (Bradaric et al., 2013), and could therefore link towards a more 'docile' temperament. Thus, should the horse demonstrate an uncharacteristic alteration in temperament by increasing in 'Docility', this may signify that there are alterations with regards to DA physiology that could indicate the development of PPID. This is highly noteworthy, as previously McFarlane (2011) suggested that observed docility was due to increased beta-endorphin circulation. In light of the findings here however, it seems probable that increased 'Docility' is a reflection instead of DA reduction. Taken together, should the owner notice elevated 'Docility' even in the absence of PPID symptomology, an in-depth endocrine work up should certainly be considered, but also proposes an interesting area for further research. 
451 Pony/cob types were found to have significantly reduced SBR, 'Anxiety' and 'Excitability' 452 scores than sports horses. In other studies (see Cabib \& Puglisi-Allegra, 2012) utilising rodents, decreased dopamine release is associated with a passive, less active stress coping style. Decreased SBR, along with lower 'Anxiety' and 'Excitability' as observed here constitutes evidence for a similar phenomenon in the horse. On the other hand, our data is somewhat at odds with the work of Lloyd et al. (2008) who observed similar 'Anxiousness' and 'Excitability' in both welsh ponies and cobs compared to Thoroughbreds and Arabs. However, Lloyd et al. (2008) formed sample groups based on breed rather than the more generalised type designation employed in this study, which may account for the discrepancy.

Interestingly, geldings scored significantly higher than mares for 'Anxiety', 'Inquisitiveness' and 'Horse-Horse Interaction'. Duberstein and Gilkeson (2010) observed that mares were more 'Anxious' than geldings, a finding directly opposing to the results presented here. Whereas Le Scolan et al. (1997) previously found no significant difference between mares and geldings with their study of equine temperament. It should be noted however, that both of these previous studies utilised lower numbers of horses ( $n=18$ and $n=72$ respectively) which may account for the lack of inter-study agreement. Given the gender imbalance often observed in some equine sporting disciplines (i.e. mares are generally favoured as polo ponies) this area is certainly worthy of further investigation.

Finally, leisure animals were found to have significantly reduced 'Anxiety' and 'Excitability' compared to those engaged in traditional sports disciplines, though had significantly elevated 'Inquisitiveness' scores than polo ponies. It could reasonably be postulated that polo ponies and sport horses are more likely to be managed under environmental conditions (i.e. housing 
and feeding regimen) that promote chronic stress potentially giving rise to the elevated 'Anxiety' and 'Excitability' along with the reduced 'Inquisitiveness' scores observed here.

\subsection{Conclusion}

This research is the first demonstration of a relationship between temperament and inferred neural DA levels within the horse. Whilst this study cannot elucidate the precise underlying mechanisms governing the DA and temperament relationship, extrapolation from previous research provides a sound basis upon which to develop future work. The ability to utilise the presentation of either increased 'Anxiety' or 'Docility' to indicate altered neural DA function at an early stage could prove valuable for the welfare of at risk animals. The potential ramifications of this work, and the group differences observed, therefore highlights this is an area that warrants further research.

\section{Acknowledgements}

The authors would like to thank the Royal Agricultural University Research Committee for the funding of this research as part of a PhD project at the Royal Agricultural University.

\section{References}

Achim, A. M., Maziade, M., Raymond, É., Olivier, D., Mérette, C. \& Roy, M. A. (2011) How prevalent are anxiety disorders in schizophrenia? A meta-analysis and critical review on a significant association. Schizophrenia Bulletin. 37(4), 811-821. 
497 Bachmann, I., Audige, L., \& Stauffacher, M. (2003) Risk factors associated with behavioural

498

499

500

501

502

503

504

505

506

507

508

509

510

511

512

513

514

515

516

517 Cabib, S. \& Bonaventura, N. (1997) Parallel Strain-Dependent Susceptibility to

518 Environmentally Induced Stereotypies and Stress-Induced behavioral Sensitization in Mice.

519 Physiology and Behavior. 61(4), 499-506.

Biggio, G., Concas, A., Corda, M. G., Giorgi, O., Sanna, E. \& Serra, M. (1990) GABAergic and dopaminergic transmission in the rat cerebral cortex: effect of stress, anxiolytic and anxiogenic drugs. Pharmacology \& Therapeutics. 48(2), 121-142.

Bradaric, Z., May, A. \& Gehlen, H. (2013) Use of the chasteberry preparation Corticosal® for the treatment of pituitary pars intermedia dysfunction in horses. Pferdeheilkunde. 29(6), 721 728.

Breier, A., Su, T.P., Saunders, R., Carson, R.E., Kolachana, B.S., De Bartolomeis, A., Weinberger, D.R., Weisenfeld, N., Malhotra, A.K., Eckelman, W.C. \& Pickar, D. (1997) Schizophrenia is associated with elevated amphetamine-induced synaptic dopamine concentrations: evidence from a novel positron emission tomography method. Proceedings of the National Academy of Sciences. 94(6), 2569-2574.

Burch. D. \& Sheerin. F. (2005) Parkinson's Disease. Lancet. 365, 622-627. 35(2), 158-163. 
521 Cabib, S., Giardino, L., Calza, L., Zanni, M., Mele, A. \& Puglisi-Allegra, S. (1998). Stress 522 promotes major changes in dopamine receptor densities within the mesoaccumbens and 523 nigrostriatal systems. Neuroscience. 84(1), 193-200.

524

525 Cabib, S. \& Puglisi-Allegra, S. (2012) The mesoaccumbens dopamine in coping with stress.

526 Neuroscience and Biobehavioral Reviews. 36, 79-89.

527

528

Cabib, S. (2006) The Neurobiology of Stereotypy II: The Role of Stress. In: Mason, G. \& 529 Rushen, J. eds. Stereotypic Animal Behaviour Fundamentals and Applications to Welfare. 2nd ed. Oxfordshire: CABI International, 2006, 227-255.

Cancela, L. M., Basso, A. M., Martijena, I. D., Capriles, N. R. \& Molina, V. A. (2001) A dopaminergic mechanism is involved in the 'anxiogenic-like'response induced by chronic amphetamine treatment: a behavioral and neurochemical study. Brain Research. 909(1), 179-186.

536

537 Cools, R. (2008) Role of dopamine in the motivational and cognitive control of behavior. The Neuroscientist. 14(4), 381-395.

539

540 Dagher, A. \& Robbins, T. W. (2009) Personality, addiction, dopamine: insights from 541 Parkinson's disease. Neuron. 61(4), 502-510. 
543 de la Mora, M.P., Gallegos-Cari, A., Arizmendi-García, Y., Marcellino, D. \& Fuxe, K. (2010)

544 Role of dopamine receptor mechanisms in the amygdaloid modulation of fear and anxiety:

545 structural and functional analysis. Progress in Neurobiology. 90(2), 198-216.

546

547 Duberstein, K. J., \& Gilkeson, J. A. (2010) Determination of sex differences in personality and 548 trainability of yearling horses utilizing a handler questionnaire. Applied Animal Behaviour 549 Science. 128(1), 57-63.

550

551 Durham, A. E., McGowan, C. M., Fey, K., Tamzali, Y. \& Kolk, J. H. (2014) Pituitary pars 552 intermedia dysfunction: Diagnosis and treatment. Equine Veterinary Education. 26(4), 216553223

554

555 Freymond, S. B., Briefer, E. F., Von Niederhäusern, R., \& Bachmann, I. (2013) Pattern of 556 social interactions after group integration: A possibility to keep stallions in group. PloS ONE. 557 8(1), e54688.

558

559 Garner, J.P. \& Mason, G.J. (2002) Evidence for a relationship between cage 560 stereotypies and behavioural disinhibition in laboratory rodents. Behavioural Brain 561 Research. 136(1), 83-92.

562

563 Graf, P., von Borstel, U. K., \& Gauly, M. (2013) Importance of personality traits in 564 horses to breeders and riders. Journal of Veterinary Behavior: Clinical Applications 565 and Research. 8(5), 316-325. 
Hausberger, M. \& Muller, C. (2002) A brief note on some possible factors involved in the reactions of horses to humans. Applied Animal Behaviour Science. 76(4), 339344.

570

571 Hemmings, A., McBride, S.D. \& Hale, C.E. (2006) Opioid circuitry and the aetiology of equine oral stereotypy. Annual Conference of the International Society of Applied 573 Ethology. January 2006, Bristol University.

Hemmings, A., McBride, S.D. \& Hale, C.E. (2007) Perseverative responding and the aetiology of equine oral stereotypy. Applied Animal Behaviour Science. 104 (1-2), 143-150.

Hori, Y., T. Ozaki, Y. Yamada, T. Tozaki, H. S. Kim, A. Takimoto, M. Endo, N. Manabe, M. 580 Inoue-Murayama. \& K. Fujita (2013) Breed Differences in Dopamine Receptor D4 Gene (DRD4) in Horses. Journal of Equine Science. 24(3), 31-36.

583 ljichi, C., Collins, L. M., Creighton, E. \& Elwood, R. W. (2013) Harnessing the power of 584 personality assessment: subjective assessment predicts behaviour in horses. Behavioural 585 Processes. 96, 47-52.

586

587 Izquierdo, I. \& Medina, J. H. (1997) Memory formation: the sequence of biochemical events 588 in the hippocampus and its connection to activity in other brain structures. Neurobiology of 589 Learning and Memory. 68(3), 285-316. 
591 Kaasinen, V., Nurmi, E., Bergman, J., Eskola, O., Solin, O., Sonninen, P. \& Rinne, J. O. 592 (2001) Personality traits and brain dopaminergic function in Parkinson's disease. 593 Proceedings of the National Academy of Sciences. 98(23), 13272-13277.

Kaminer, J., Powers, A.S., Horn, K.G., Hui, C. \& Evinger, C. (2011) Characterizing 596 the Spontaneous Blink Generator: An Animal Model. The Journal of Neuroscience. 31(31), 11256-11267.

Kienast, T., Hariri, A.R., Schlagenhauf, F., Wrase, J., Sterzer, P., Buchholz, H.G., Smolka, M.N., Gründer, G., Cumming, P., Kumakura, Y. \& Bartenstein, P. (2008) Dopamine in amygdala gates limbic processing of aversive stimuli in humans. Nature Neuroscience. $11(12), 1381-1382$.

603

604

Kilgour, R. (1975) The open-field test as an assessment of the temperament of dairy cows. Animal Behaviour. 23, 615-624.

606

Lansade, L. \& Simon, F. (2010) Horses' learning performances are under the influence of several temperamental dimensions. Applied Animal Behaviour Science. 125(1), 30-37.

Lansade, L., Coutureau, E., Marchand, A., Baranger, G., Valenchon, M. \& Calandreau, L. (2013) Dimensions of Temperament Modulate Cue-Controlled Behavior: A Study on

612 Pavlovian to Instrumental Transfer in Horses (Equus Caballus). PLoS ONE. 8(6): e64853. 
614 Le Scolan, N., Hausberger, M., \& Wolff, A. (1997) Stability over situations in temperamental 615 traits of horses as revealed by experimental and scoring approaches. Behavioural Processes. $616 \quad 41(3), 257-266$.

617

618 Lloyd, A. S., Martin, J. E., Bornett-Gauci, H. L. I. \& Wilkinson, R. G. (2007) Evaluation of a 619 novel method of horse personality assessment: Rater-agreement and links to behaviour. Applied Animal Behaviour Science. 105(1), 205-222.

621

Lloyd, A. S., Martin, J. E., Bornett-Gauci, H. L. I. \& Wilkinson, R. G. (2008) Horse personality: variation between breeds. Applied Animal Behaviour Science. 112(3), 369-383.

624

McAfee, L.M., Mills, D.S. \& Cooper, J.J. (2002) The use of mirrors for the control of stereotypic weaving behaviour in the stabled horse. Applied Animal Behaviour Science. 78(2), 159-173.

McBride, S. \& Hemmings, A. (2005) Altered mesoaccumbens and nigro-striatal dopamine physiology is associated with stereotypy development in a non-rodent species. Behavioural Brain Research. 159,113-118.

McBride, S.D. \& Hemmings, A. (2004) Causal factors of equine stereotypy. In:

634 Alliston, J., Chadd, S., Ede, A., Longland, A., Moore-Colyer, M., Hemmings, A. \& Hyslop, J. eds. Emerging Equine Science. Nottingham: Nottingham University Press. 2004, 35-65. 
638 McCullough, L. D., \& Salamone, J. D. (1992). Anxiogenic drugs beta-CCE and FG 7142

639

640

641

642

643

644

645

646

647

648

649

650

651

652

653

654

655

656

657

658

659

660

\section{7}

increase extracellular dopamine levels in nucleus accumbens. Psychopharmacology. 109(3), 379-382.

McFarlane, D. (2011) Equine pituitary pars intermedia dysfunction. Veterinary Clinics of North America: Equine Practice. 27(1), 93-113.

McFarlane, D., Dybdal, N., Donaldson, M. T., Miller, L. \& Cribb, A. E. (2005) Nitration and Increased a-Synuclein Expression Associated With Dopaminergic Neurodegeneration In Equine Pituitary Pars Intermedia Dysfunction. Journal of Neuroendocrinology. 17(2), 73-80.

McFarlane, D., Paradis, M. R., Zimmel, D., Sykes, B., Brorsen, B. W., Sanchez, A. \& Vainio, K. (2011) The Effect of Geographic Location, Breed, and Pituitary Dysfunction on Seasonal Adrenocorticotropin and $\alpha$-Melanocyte-Stimulating Hormone Plasma Concentrations in Horses. Journal of Veterinary Internal Medicine. 25(4), 872-881.

McGrogan, C., Hutchison, M. D. \& King, J. E. (2008) Dimensions of horse personality based on owner and trainer supplied personality traits. Applied Animal Behaviour Science. 113(1), 206-214.

Menza, M. (2000) The personality associated with Parkinson's disease. Current Psychiatry Reports. 2(5), 421-426. 
661 Momozawa, Y., Kusunose, R., Kikusui, T., Takeuchi, Y. \& Mori, Y. (2005a) Assessment of equine temperament questionnaire by comparing factor structure between two separate surveys. Applied Animal Behaviour Science. 92(1), 77-84.

664

Momozawa, Y., Ono, T., Sato, F., Kikusui, T., Takeuchi, Y., Mori, Y. \& Kusunose, R. (2003)

Assessment of equine temperament by a questionnaire survey to caretakers and evaluation

667

of its reliability by simultaneous behavior test. Applied Animal Behaviour Science. 84(2), 127-

668 138.

669

670

Momozawa, Y., Takeuchi, Y., Kusunose, R., Kikusui, T. \& Mori, Y. (2005b) Association

671

between equine temperament and polymorphisms in dopamine D4 receptor gene.

Mammalian Genome. 16(7), 538-544.

673

Nagy, K., Bodo, G., Bárdos, G., Banszky, N. \& Kabai, P. (2010) Differences in temperament traits between crib-biting and control horses. Applied Animal Behaviour Science. 122(1), 4147.

677

678

Nikulina, E. M., Miczek, K. A. \& Hammer, R. P. (2005) Prolonged effects of repeated social defeat stress on mRNA expression and function of $\mu$-opioid receptors in the ventral tegmental area of rats. Neuropsychopharmacology. 30(6), 1096-1103.

681

682 Ninomiya, S., Sato, S. \& Sugawara, K. (2007) Weaving in stabled horses and its relationship 683 to other behavioral traits. Applied Animal Behaviour Science. 106(1), 134-143. 
685 Northey, G. (2003) Equestrian injuries in New Zealand, 1993-2001: knowledge and experience. The New Zealand Medical Journal (Online). 116(1182).

687

Pallanti, S., Quercioli, L. \& Hollander, E. (2014) Social anxiety in outpatients with schizophrenia: a relevant cause of disability. American Journal of Psychiatry. 161(1), 53-58.

690

691 Park, I.J., Jung, D.C., Hwang, S.S.H., Jung, H.Y., Yoon, J.S., Kim, C.E., Ahn, Y.M. \& Kim, 692 Y.S., (2016) Longitudinal relationship between Personal and Social Performance (PSP) and anxiety symptoms in schizophrenia. Journal of Affective Disorders. 190, 12-18.

Parker, M., McBride, S.D., Redhead, E.S. \& Goodwin, D. (2009) Differential place and response learning in horses displaying an oral stereotypy. Behavioural Brain Research. 200,100-105.

698

699

Randle, H.D. (2015) Personality and performance: the influence of behaviour. In:

700

Williams, J.M. \& Evans, D. eds. Training for equestrian performance. Wageningen:

701 Wageningen Academic Publishers. 2015, 301-320.

702

703

Roberts, K., Hemmings, A., Moore-Colyer, M. \& Hale, C. (2015) Cognitive differences

704 in horses performing locomotor versus oral stereotypic behaviour. Applied Animal

705 Behaviour Science. 168, 37-44. 
707 Roebel, A.M. \& MacLean, W.E. (2007) Spontaneous eye-blinking and stereotyped behavior in older persons with mental retardation. Research in Developmental

711 Seaman, S. C., Davidson, H. P. B., \& Waran, N. K. (2002) How reliable is temperament assessment in the domestic horse (Equus caballus)? Applied Animal Behaviour Science. 78(2), 175-191.

Seger, C. A. \& Cincotta, C. M. (2006). Dynamics of frontal, striatal, and hippocampal systems during rule learning. Cerebral Cortex. 16(11), 1546-1555.

Stur, I. (1987) Genetic aspects of temperament and behavior in dogs. Journal of Small Animal Practice. 28(11), 957-964.

720 MPTP-Treated Monkeys. Experimental Neurology. 158, 214-220.

Todes, C. J. \& Lees, A. J. (1985) The pre-morbid personality of patients with Parkinson's disease. Journal of Neurology, Neurosurgery \& Psychiatry. 48(2), 97-100.

728 Valenchon, M., Lévy, F., Prunier, A., Moussu, C., Calandreau, L. \& Lansade, L. (2013) Stress Modulates Instrumental Learning Performances in Horses (Equus caballus) in Interaction with Temperament. PLoS ONE. 8(4): e62324. 
732 Visser, E. K., Van Reenen, C. G., Blokhuis, M. Z., Morgan, E. K. M., Hassmén, P., Rundgren, 733 T. M. M. \& Blokhuis, H. J. (2008) Does horse temperament influence horse-rider 734 cooperation? Journal of Applied Animal Welfare Science. 11(3), 267-284.

735

736 Visser, E. K., Van Reenen, C. G., Rundgren, M., Zetterqvist, M., Morgan, K. \& Blokhuis, H. 737 J. (2003) Responses of horses in behavioral tests correlate with temperament assessed by 738 riders. Equine Veterinary Journal. 35(2), 176-183. 